Barberà, Gemma. 2014. Use and functions of spatial planes

in Catalan Sign Language (LSC) discourse. Sign Language Studies 14 (2)

\title{
Use and functions of spatial planes \\ in Catalan Sign Language (LSC) discourse
}

\author{
Gemma Barberà ${ }^{1}$ \\ UMR7023 (CNRS \& Paris 8) \\ 59 rue Pouchet, Paris 75017 (France) \\ gemma.barbera@gmail.com \\ gemmabarbera.wordpress.com
}

\footnotetext{
${ }^{1}$ Gemma Barberà is a postdoctoral researcher at UMR7023 (CNRS \& Paris 8) specialising on impersonal reference in sign language. Her dissertation, defended in 2012 at Universitat Pompeu Fabra (Barcelona), deals with reference and more specifically with definiteness and specificity marking in Catalan Sign Language discourse.
} 
Abstract. While the phonological description of spatial planes which configure signing space is very detailed (Brentari 1998; Liddell and Johnson 1989; Sandler 1989), our knowledge of its use and functions at the discourse level is still very limited. This article aims at offering a description of the use of the spatial planes in Catalan Sign Language (LSC). It provides a unified treatment of different linguistic phenomena that have been hitherto described separately in the sign language literature, namely hierarchical relations, locatives, as well as other aspects that have not yet been the subject of research neither in LSC nor in other signed languages, such as the encoding of specificity. As shown, the features found in two of the three spatial planes as described in the phonological literature are also relevant beyond the sentence level and they serve distinctive discourse functions.

Key words: Catalan Sign Language (LSC), contrastive topics, discourse, hierarchical relations, locatives, signing space, spatial planes, specificity

\section{Introduction}

When we look at a signed conversation, the most striking aspect that we may find is that sign languages (SLs) use space for the representation of meaning. While spoken languages use the audio-vocal modality, SLs use the visual-spatial modality. As a consequence of this modality, signing space, which is the three dimensional space in front of the signer's body, is thoroughly used. Linguistic expressions in SLs rely on signing space and the different components of the grammar show dependence on it. At the phonological level, space is used contrastively in the place of articulation parameter of signs (Brentari 1998; Sandler 1989). At the morphosyntactic level, signs are modulated in space for grammatical purposes to express number, person, as well as the arguments of the verb (Janis 1992; Mathur 2000; Padden 1988). As far as the discourse level is concerned, spatial locations are associated with discourse referents (Klima and Bellugi 1979).

Nevertheless, the analysis of space in SLs is not free of controversy and it has been, and still is, a matter of debate among SL researchers. Signs directed to space may externally be seen as very similar to co-speech gesture and this has led some researchers 
to argue that signs which obligatorily use locations in space (such as pronouns and verb agreement) are composed of a linguistic and a gestural part (Liddell 1990, 2003; Meier 1990). This implies that locations are analysed as a gradient continuum and very rarely as categorical elements. In an opposite view, the referential locus view maintains that spatial locations denote a formal relationship between referent and location for further use later in the discourse (Friedman 1975; Kegl 1976/2003; Lillo-Martin and Klima 1991; Neidle et al. 2000). This view considers that although it is generally assumed in the syntactic literature that Noun Phrases (NPs) are considered to contain abstract referential features, SLs show the overt morphological expression of referential distinctions through association of distinct referents with specific spatial locations. Hence, locations in space are analysed as the overt manifestation of referential indices.

This article argues that Catalan Sign Language (llengua de signes catalana, LSC) shows a systematic usage of the syntactic use of two of the three spatial planes, namely the horizontal and the frontal planes. On the basis of small-scale corpus data, I provide a description of how these two spatial planes are used in discourse. I concentrate only on the so-called syntactic use of space, leaving the topographic one, and even more interestingly the interaction between these two, for future research. A categorical division of a three-dimensional area such as signing space into different directions is available, due to the systematic use of spatial planes in LSC with respect to their function in discourse. As shown by the insights into the ongoing debate over the nature of space in the signed modality in terms of gestural and linguistic properties, this article recognises particular uses of spatial planes to express discourse categories, which contributes to the characterisation of the abstract import of signing space.

The small-scale corpus used for the present study includes data from seven native deaf signers, three women and four men, aged between 41 and 62 years old and living in the area of Barcelona. The small-scale LSC corpus comprises so far about 5,108 signs and it is a composite of genres, such as news, interviews, documentaries, tales, as well as different discourse modes, namely narrative, explicative, and dialogue. It consists of three types of data, namely semi-spontaneous discourses, videos recorded for other purposes, and elicited data (see Barberà 2012, for a complete description of the data set).

The rest of the article is organised as follows. Section 2 offers a thorough description of the use of signing space beyond the sentence level. It also presents the 
spatial morpheme, and it reviews the two main spatial functions. Section 3 introduces a description of the three spatial planes and the features contained within non-descriptive locations. Section 4 is devoted to the analysis of use and functions of spatial planes. Section 5 summarises the main findings.

\section{Signing space}

The actual space where the articulations of signs take place is called signing space. It is considered to be constrained to the horizontal and the frontal plane in front of the signer's torso (Figure 1). ${ }^{2}$ The body of the signer itself is also used as a possible location for the articulation of signs. As argued by Klima and Bellugi (1979), signing space is not only used for articulatory reasons where the hands and the arms can move toward but, more importantly, it carries linguistic meaning.

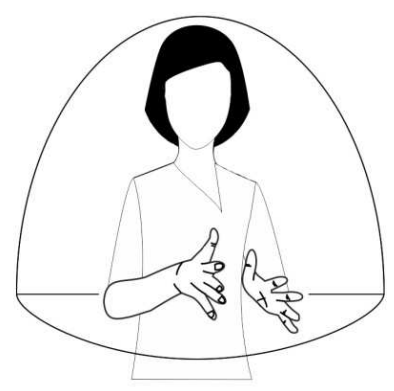

Figure 1. Signing space

\subsection{Beyond the sentence}

Discourse referents (DRs) in SLs are associated with spatial locations, which may be further referred back to in coreferential contexts (Klima and Bellugi 1979). Such spatial location is called "referential locus" or "r-locus" (Lillo-Martin and Klima 1990) and it may be established across sentence boundaries. Such an example is shown in (1) where the signer associates the nominal "son" with a spatial location by means of directing an index sign to the contralateral area (i.e. the left area in a right-handed signer, see section

\footnotetext{
${ }^{2}$ The description of space given in this article focuses only on LSC, which shares many features with other Western urban sign languages. However, non-urban SLs are more and more studied in different corners of the world. As described so far, these non-urban SLs show differences in the grammar and use of space. For instance, Kata Kolok, a village sign language in North Bali, uses a much larger signing space that goes beyond the limits described here (Marsaja 2008; de Vos 2012).
} 
3.1). In the second clause in (1), two index signs are directed to the same locus and the coincidence of the direction triggers a coreferential interpretation. ${ }^{3}$

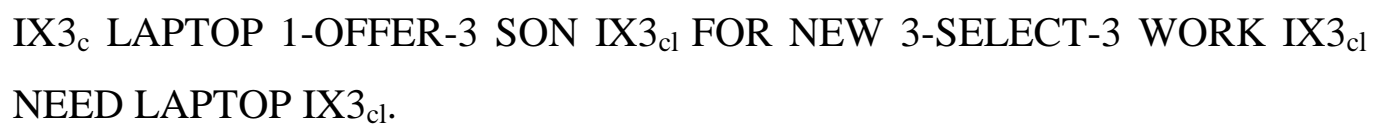

'I will offer this laptop to my son, because he has been selected for a new job and he needs one.'

The following figure contains a sequence of the two index signs appearing in (1). The stills in Figure 2a correspond to the introduction and the spatial association of the DR SON. Figure $2 b$, corresponding to an index sign in the second sentence in (1), shows that further mentions of the DR are realised with a pronominal index sign pointing to the same direction on the horizontal plane previously established.

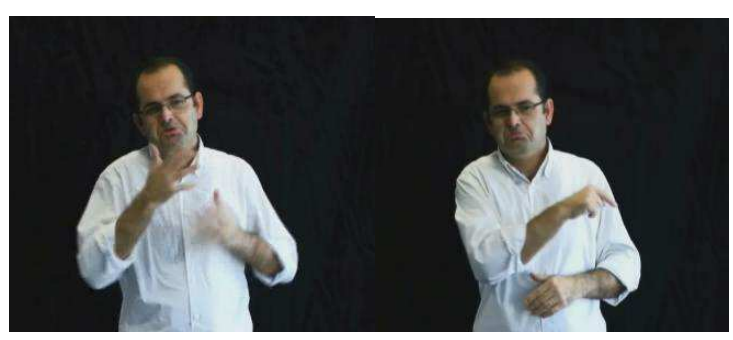

a. SON IX3

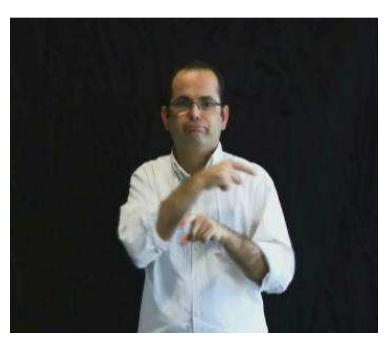

b. IX3

Figure 2. First and further mention of a localised discourse referent

The set of linguistic mechanisms used to both establish a DR in space and to refer back to it have been described for specific SLs, namely American Sign Language (Winston 1995), British Sign Language (Morgan 1996), and LSC (Barberà 2012). The most commonly used mechanisms are index signs (the so-called "pointing signs"), agreeing verbs, weak hand in dominance reversals sequences, body lean, and classifier handshapes. These mechanisms not only establish a DR in signing space but they also contribute to keeping the referent active in the discourse and constitute the strategies for reference-tracking. Although the association between DRs and signing space is a typical

\footnotetext{
${ }^{3}$ The usual glossing conventions in the sign language literature are followed, according to which manual signs are represented by the capitalized word corresponding to the translation of the sign. The relevant abbreviations here are the following: IX3 (index sign); \#-VERB-\# (verb agreeing with subject and object: the numbers refer to the grammatical person); subindices mark direction towards signing space: 1 (low), $\mathrm{u}$ (up), ip (ipsilateral); cl (contralateral); ce (centre). A line above the glosses indicates the scope of nonmanuals: eg (eyegaze); br (brow raised). Reduplication is indicated with +++ .
} 
characteristic of SLs and this spatial use may seem a unique and differential aspect of SLs due to their visual-spatial modality, the interpretation and resolution of coreferential relations do not differ across language modalities (Emmorey 2007).

\subsection{Spatial morpheme}

Some authors have argued for a phonemic/phonetic distinction in the direction that index signs may take because they are directed to a three-dimensional extension and therefore the value for an r-locus is difficult to categorise (Janis 1992; Kooij 2002). While the phonology of index signs is considered to be an abstract point in space, their phonetics is the actual direction, and thus the broader dimension, they may get. The different directions that an index sign towards space may have are in fact considered a gradient property, which can be compared to the opening of vocals in spoken languages (Rathmann and Mathur 2002; Russell and Janzen 2008). In the syntax-discourse interface, the challenge with r-loci is that researchers have focused primarily on the physical point in space where index signs are directed to, neglecting the fact that what matters is not the exact point but rather its categorical interpretation in the linguistic system.

Following Wilbur (2008), I consider that the spatial direction where index signs can be directed to consists in an abstract and unique point in space. What matters is not the exact area, but rather the abstract end point that is expressed with the localisation of signs which is interpreted in the grammar as a categorical element, rather than as a gradient one. The relevance of Wilbur's account is that she makes precise that what it is important is that the spatial point is categorically defined and interpreted within the linguistic system. Moreover, this article shows that the abstract point may be established on different areas from the spatial planes, which are categorically distinguished. Thus a unique spatial morpheme exists in the grammar of the language, which consists in an abstract point where indexical signs and other localisation mechanisms are oriented to and established on different categorical areas within spatial planes.

\subsection{Spatial functions}

Since the beginnings of SL linguistics research, it has been argued that space undertakes two functions, namely a syntactic and a topographic one (Poizner, Klima and Bellugi 1987). The syntactic function is an abstract use of space in which DRs are arbitrarily 
localised to identify the arguments of the verb. A particular r-locus is assigned to a DR, which is movable as it can be shifted without affecting the truth conditions of the sentence. The topographic function, in contrast, is used to express spatial relations among objects and it is represented by meaningful locations that exploit the iconic properties of the visual-spatial modality. Topographic loci are meaningful by themselves, so a small change in the location affects its truth conditions. In this latter case, space is used to represent spatial arrangements via signed descriptions and the spatial relations of signs are significant. To avoid implying that the topographic use of space is non-syntactic, I call the localisations occurring in the topographic use of space "descriptive", and the ones that occur in the syntactic use of space, "non-descriptive", following Quer et al. (2005). The descriptive localisation in Figure 3a represents a bike leaning against a tree. Figure $3 \mathrm{~b}$ represents a person seated on a tree. They are motivated by a mapping with the situation described and they are represented by meaningful loci.

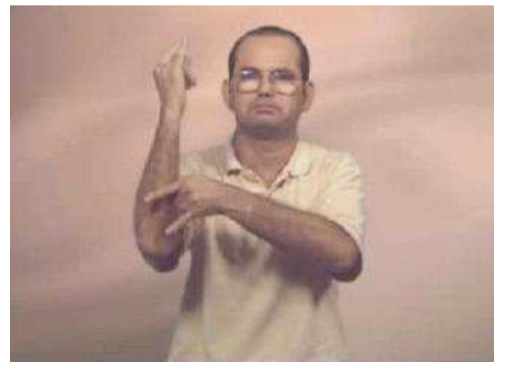

a. Bike leaning against a tree

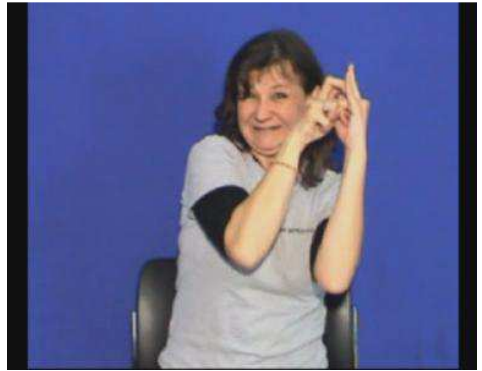

b. Person seated on a tree

Figure 3. Descriptive localisation

This contrasts with non-descriptive localisations which are arbitrarily and abstractly established, as previously shown in Figure 2. Comparing descriptive and nondescriptive localisation shows that descriptions of spatial layout use the same horizontal plane of signing space as do SL nominals, pronominal reference and verb agreement devices. Both functions make use of r-loci, the difference being in its significance: loci in signed descriptions are meaningful because they represent actual spatial topographical relations, whereas abstract loci are not meaningful by themselves because they are established for syntactic and discourse purposes.

According to some works, non-descriptive localisations consist in conceptualisations of spatial relations of objects, which are conceptualised under some 
frame of reference. As the works by Arrk (2009) and Perniss (2007) show, different sign languages make use of different perspectives and frames of reference. ${ }^{4}$ The main difference between descriptive and non-descriptive localisations from an interpretation and analytical level is that the former conceptualise the position of the object in signing space and the latter establish a formal and abstract relationship between the object and the corresponding associated r-locus. Moreover, they also present another difference at the form level: while descriptive uses of space exploit richer and freer sets of r-loci in the three-dimensional space, non-descriptive ones are composed of spatial planes and fixed trajectories within each plane. Importantly, non-descriptive r-loci are not restricted to the horizontal plane in front of the signer as originally argued by Klima and Bellugi (1979). Some authors note that nouns can also be assigned r-loci vertically above or below the horizontal plane in certain circumstances (Fischer and Gough 1978; MoralesLópez et al. 2005; Schlenker and Lamberton 2012; Shepard-Kegl 1985).

A detailed phonological analysis of locations and especially a thorough description of body locations for the production of signs have been provided (Brentari 1998; Liddell and Johnson 1989; Sandler 1989). Locations occur on three planes projected with respect to the signer's body (Brentari 1998, 120). First, the horizontal plane stands perpendicularly to the body of the signer and it is the default plane where the majority of signs are localised (Figure 4a). Second, the frontal plane is defined by all those points included on the plane in parallel to the body (Figure 4b). Finally, the midsagittal plane is vertically perpendicular to the body of the signer (Figure 4c).

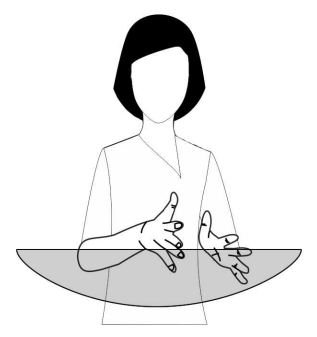

a. Horizontal

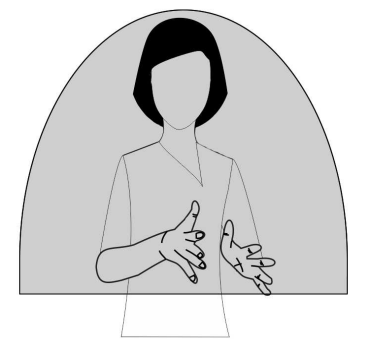

b. Frontal

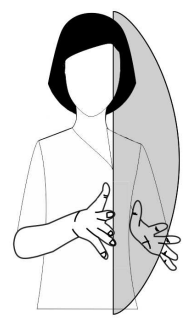

c. Midsagittal

Figure 4. Spatial planes

\footnotetext{
${ }^{4}$ It is interesting to note that some village SLs have been described as making extensive use of descriptive localisations. Kata Kolok SL predominantly employs topographical space (Marsaja 2008; de Vos 2012). Signers use real-world locations instead of establishing abstract locations, despite the ambiguities (e.g. the sign for a place may be localised differently depending on where the signer is in relation to the referent). Kata Kolok uses an absolute frame of reference (Levinson 1996), which is used to a lesser extent in western SLs.
} 
However, when it comes to analyse space as used in discourse, the description is not as detailed as it is at the lexical level. The authors agree that the syntax-discourse interface determines the position of the sign in space and they postulate some major spatial areas interfacing with the discourse domain. Non-descriptive locations, which are the main topic of this article, are constrained within these three planes. The following section is devoted to the description of each plane and the features contained within.

\section{Spatial planes and features}

Non-descriptive locations are used to identify the arguments of the verb, which are arbitrarily localised. They are categorically defined as occurring in the three spatial planes projected with respect to the body of the signer. The current description of the planes is based on Brentari (1998), and the major features distinctions are presented according to the phonological account in Liddell and Johnson (1989) and Sandler (1989). To the best of my knowledge, no previous work has been done on the application and extension of the spatial planes and their features in discourse use.

The geometrical units in which space may be divided into are points, axes and planes. Points are zero-dimensional elements which intersect with the three spatial planes. Axes are one-dimensional lines which consist in a set of points whose coordinates satisfy a given linear equation. Finally, planes are sets of points which extend in a two-dimensional area. Although points and axes have been used to analyse pronominal and agreement verbs in signing space (Padden et al. 2010; Wilbur 2008), this article uses the notion of spatial plane because it allows to focus on the different features contained within a two-dimensional area. The features established on each plane are the result of a particular direction of index signs or other localisation mechanisms. What matters is not the particular point in space, but rather the area in a plane that gets activated through the direction articulated with the index sign, as already explained in section 2.2 .

\subsection{Horizontal plane}

The horizontal plane stands perpendicularly to the body of the signer and since the beginning of SL linguistics research it is commonly considered the default plane where the majority of signs are localised (Klima and Bellugi 1979). According to Sandler 
(1989), the horizontal plane can be divided into [ipsilateral] and [contralateral]. In Liddell and Johnson (1989)'s model, the horizontal plane is further divided into a [centre] feature. Following the latter work, the features are distinguished in accordance with the signer's body: [centre] is in line with the breast; [ipsilateral] is in line with the outside edge of the dominant shoulder, and [contralateral] aligns with the non-dominant shoulder. Figure 5 is an example of the divisions within the horizontal plane for a righthanded signer, in which the ipsilateral part coincides with the right hand part. This tripartite distinction is the one found in LSC and a DR may be associated with an $\mathrm{r}$ locus established in one of these three areas.

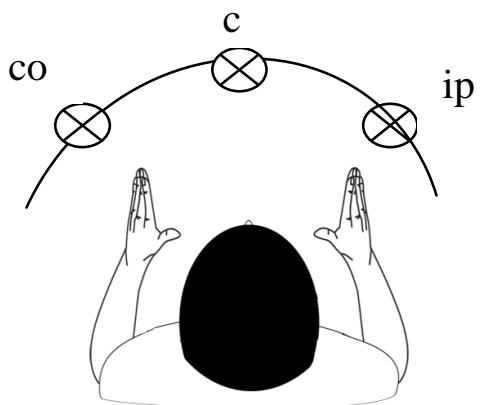

Figure 5. Horizontal plane

The features within the horizontal plane are grammatically relevant in the expression of plurality, temporality and aspect. As for plurality, signs directed towards the axis which unifies the contralateral and the ipsilateral part and repeated up to three times denote reduplication. This same axis also expresses sequences of temporal units in the anaphoric axis, which is used to establish events with respect to a temporal point of reference (Brennan 1983; Engberg-Pedersen 1993). Moreover, the sequential temporal axis also makes use of this plane to localise the different temporal events or nominals from the contralateral to the ipsilateral part. As for aspectual information, distributive aspect for example is produced by repeating the sign at different areas on the horizontal plane without any formational changes.

\subsection{Frontal}

The frontal plane, according to Brentari (1998)'s terminology, extends vertically to the body of the signer. The features [lower] and [upper] described by Sandler (1989) are clearly distinguished when the shoulder and the head are considered. The space from the 
height of the shoulder and upwards is considered to be the upper part. The lower part extends below the height of the shoulder.

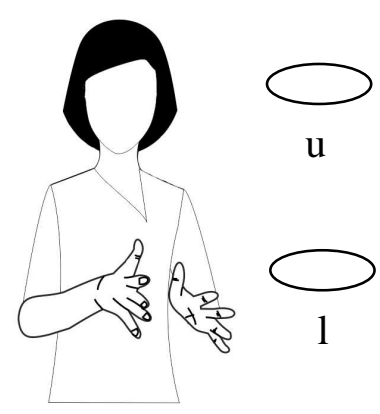

Figure 6. Frontal plane

In LSC the lower part of the frontal plane is the default area where an r-locus is established (which coincides with the lateral part of the horizontal plane). Interestingly, the upper part is also used to associate DRs, but this upper establishment arises a marked meaning, as will be shown in section 4.2.

\subsection{Midsagittal}

The midsagittal plane extends vertically and perpendicularly to the body of the signer. Two features are associated with it: "[proximal] is defined as a distance a few inches from the specified place, and [distal] is a comfortable arm's length away from the place" (Sandler 1989, 136). The distinction between the two features is thus established in accordance with the angle the elbow forms: the [proximal] feature is triggered when the angle of the elbow is smaller than $90^{\circ}$, and the [distal] feature when the angle is bigger than $90^{\circ}$.

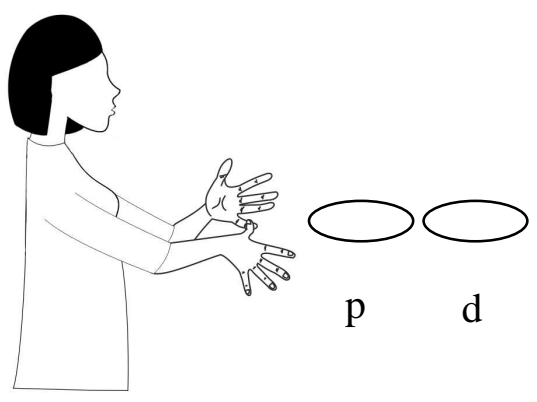

Figure 7. Midsagittal plane 
The features [proximal] and [distal] unify the axis used to express temporal information, where present tense is signed in the proximal area, and future tense is signed in the distal area. They are also relevant at the lexical level. In LSC, the sign for TOMORROW is signed in the proximal area, and the sign for THE-DAY-AFTERTOMORROW is signed in the distal area. This axis also forms the mixed temporal axis (Engberg-Pedersen 1993) which conflates the anaphoric and the deictic axis. That is to say, the temporal information in this axis is marked in the discourse and anchored in the context, and lexical signs like FROM-NOW-ONWARDS and UNTIL-NOW are realised starting in the proximal area and moving towards a distal area with respect to the signer's body.

This article mainly focuses on non-descriptive r-loci established on the three spatial planes just presented. However, locations can also occur as body-anchored forms, since the body of the signer and the spatial area immediately in front of it is also considered to be part of signing space. In such contexts the signer's body is used as the location parameter. Body-anchored locations are realised with verbs agreeing with the body of the signer (generally assigned to first person). Importantly, they need to be distinguished from signs which have an internal phonological specification where the location parameter is phonologically specified at a determined location closer or in contact with the body of the signer. Since the final goal here is to describe how locations in signing space work at the discourse level, I will leave body-anchored locations for future research.

\section{Use and functions}

In this section, the areas within spatial planes are presented with respect to their discourse function in non-descriptive contexts in LSC. A subsection is devoted to each plane.

\subsection{Horizontal plane: Contrast}

\subsubsection{Kinds of spatial entities}

In LSC, not all the DRs are equally localised in signing space and there is a restriction of the kinds of entities that occupy an r-locus within the horizontal plane. The motivation for the restriction in the kind of entities localised is found in ontological classes. Reference to third (person) entities identifies the thing the conversation is about 
by locating it with reference to the spatio-temporal location of the event. LSC shows a distinction with respect to localisation on the horizontal plane between the expression of DRs on the one hand, and the expression of other classes which include facts, propositions, and events, on the other. When third (person) DRs are mentioned, the LSC index sign is directed towards the lateral sides of signing space (i.e. ipsilateral and contralateral). In contrast, when an index sign refers to a fact, proposition or event, the index sign is directed to the centre area. For the sake of simplicity, I call this second cluster of classes "non-entities". In this informal description, I define non-entities as being negatively identified with respect to DRs. DRs are entities denoting the object of thought or the thing the conversation is about, which map linguistic expressions to constructs that are built along the discourse progression (Karttunen 1976). According to our data, the areas on the horizontal plane are specialised: DRs are associated with an $\mathrm{r}$ locus on the lateral parts and non-entities with an r-locus in the central part. A further distinction between DRs and non-entities is found in its anaphoric behaviour. Unlike DRs, which are localised during first mention and can be picked up by distant and nondistant resumptive pronouns, non-entities are never localised during first mention, but they are rather introduced into the discourse without being spatially established. However, they may have non-distant anaphoric pronouns referring back to them. Although being characterised as non-entities, they can also serve as antecedents towards which resumptive pronouns refer back to (for instance, in the case of propositions). Distant resumptive pronouns linked to non-entities have not been found in the smallscale corpus. An example of a localised entity is (1), repeated here for convenience as (2) with a subsequent utterance added, which contains a non-distant and a distant pronoun, marked in boldface below.

\footnotetext{
IX3 $3_{\mathrm{c}}$ LAPTOP 1-OFFER-3 SON IX3 $3_{\mathrm{cl}}$ FOR NEW 3-SELECT-3 WORK IX3 $\mathbf{3}_{\mathrm{cl}}$ NEED LAPTOP IX $\mathbf{3}_{\mathrm{cl}}$. [...] IX1 SURE IX $\mathbf{3}_{\mathrm{cl}}$ HAPPY.

'I will offer this laptop to my son, because he has been selected for a new job and he needs one. [...] I'm sure he will be very happy.'
}

(2) contrasts with the following example in (3) where a non-entity is introduced although not localised. However, in the subsequent sentence, a resumptive pronoun which consists in a lax pointing directed to the centre is used. In (3) the non-entity of 
Hitler becoming the German chancellor is not established in space. But a non-distant resumptive pronoun refers back to the just introduced non-entity (i.e. the proposition). This is realised with a lax pointing sign directed to the centre, as graphically shown in Figure 8.

\section{YEAR 1933 HITLER PERSON-3 ip START 1-APPOINT-3 ip $_{\text {EQUAL/SAME }}$} RESPONSIBLE MAXIMUM GERMANY ZONE. IX3 $\mathbf{3}_{\mathbf{c}}$ NOVELTY LAW

'In 1933 Hitler was appointed chancellor of Germany.

This (issue) entailed the creation of a new law.'

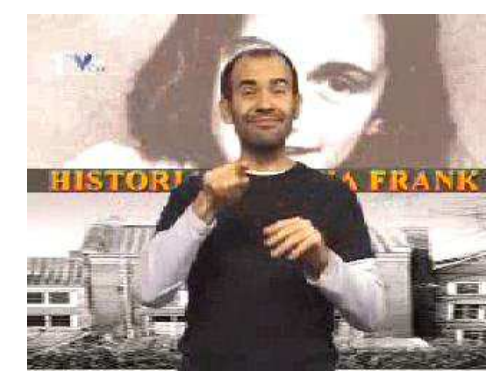

Figure 8. Coreferential index referring to a non-entity in LSC

A clear distinction between the entity-like properties of the lateral parts of LSC signing space and the non-entity-like properties of the central part is present in the use of the horizontal plane in LSC.

\subsubsection{Contrastive topics}

In LSC the two features on the horizontal plane used to localise DRs are not grammatically relevant themselves. Whether an r-locus is precisely established on the ipsilateral or on the contralateral part does not mark any difference on the grammar of LSC. The interpretation of (4a) and (4b) is equivalent regardless of the lateral area where each NP is localised. As the translation shows, the denotation of the nominal is not affected by the localisation side.
a. YESTERDAY JOAN ${ }_{\text {ip }} 3_{\text {ip }}$-TELL- 1 PILAR IX $3_{\text {cl }}$ SICK
b. YESTERDAY JOAN ${ }_{\mathrm{cl}} 3_{\mathrm{cl}}$-TELL-1 PILAR IX $3_{\text {ip }}$ SICK

'Yesterday Joan told me that Pilar was sick.' 
In LSC the main motivations forcing the localisation of a DR on the two side parts are due to assimilation processes and economy reasons, which escape the grammatical restrictions of the language. However, when both the ipsilateral and the contralateral parts are used in the same fragment of discourse to localise two DRs, a contrastive relation arises. This is an overt marking of the expression of contrastive topics (Büring 2003; Vallduví and Vilkuna 1998, for contrast in the spoken language literature; Wilbur 2012, for a general overview of signed languages). Engberg-Pedersen (1993, 74) descriptively defines this use as a convention of comparison, used when two DRs need to be compared or contrasted. In LSC, this contrastive use of the lateral parts coincides with double contrast as defined in Mayol (2010). That is, two clause discourses in which two DRs are introduced in each clause and their respective verbs predicate two different, contrasting actions. The opposed r-loci distinguish the two DRs and are interpreted as contrastive topics. Signing space is then restricted to the two variables and references to one or the other will be represented by a direction of the index sign to the two opposed lateral parts on the horizontal plane, as shown in Figure 9.

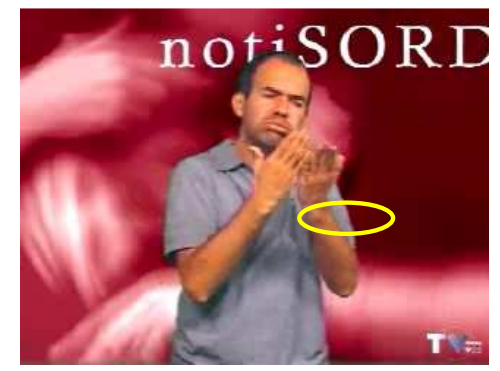

a. Contralateral r-locus for referent ${ }_{x}$

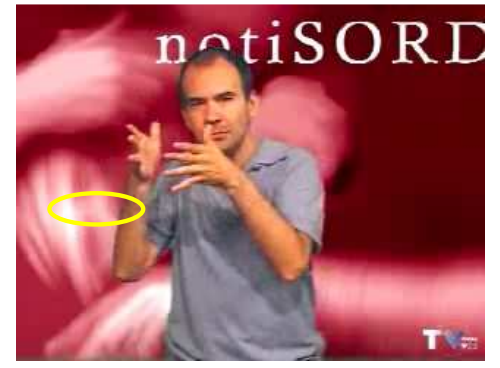

b. Ipsilateral r-locus for referent $y$

Figure 9. Contrastive r-loci

Such an example is shown in (5) where the two DRs, 'Francesc' and 'Joana' are localised in the ispliateral and the contralateral part, respectively. For each one a different predicate is expressed and the double contrast is overtly expressed with the establishment of the two r-loci.

\footnotetext{
br

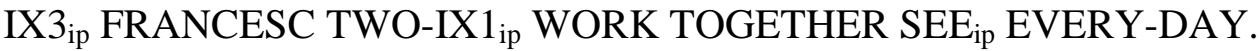
br

IX $3_{\mathrm{cl}}$ JOANA WORK SCHOOL ANOTHER $_{\mathrm{cl}}$, TWO-IX1 $1_{\mathrm{cl}} \mathrm{SEE}_{\mathrm{cl}} \mathrm{ONE}_{\mathrm{u}}$.
} 
'As for Francesc, we work together and we see each other every day.

But Joana works at another school and we only see each other once in a while.'

Unless the DR is reintroduced by the nominal, the distinction ipsilateral-contralateral is kept throughout the discourse as long as there is no shift in the frame of reference.

\subsection{Frontal plane: Bundle of meanings}

The frontal plane in LSC is distinguished between the [upper] and the [lower] features. The lower part is the default area where DRs are generally localised. The upper part is a marked area, which includes a bundle of particular meanings. Morphophonologically, the feature [upper] is instantiated with an homomorph morpheme which denotes four particular meanings. Homomorphs are morphemes with the same form but different meaning. An English example is the morpheme -er which can denote comparative meaning, as in bigger; human agentivity, as in teacher, and inanimate instrument, as in screwdriver. In the case of LSC, the homomorph represented by the feature [upper] is specialised with four meanings, indicated in (6). Below a subsection is devoted to each one.

(6) [upper]: \{hierarchical position, locative information, non-specificity, absence in the physical context

\subsubsection{Hierarchical relations}

The upper part of the frontal plane is used to denote social hierarchical relations, and more particularly superiority. The contrast between upper and lower frontal plane is associated with asymmetrical relations such as parents-children, boss-worker, professorstudent, etc. In such contexts, an r-locus established on the upper part of the frontal plane denotes the individual who is higher in the social hierarchy. This use has been previously described for LSC (Morales-López et al. 2005), for Indo-Pakistani Sign Language (Zeshan 2000), and for ASL (Liddell 1990; Schlenker and Lamberton 2012). Definite NPs formed by common nouns such as MINISTRY, GOVERNMENT (Figure 10), BOSS, DEAN, FATHER^MOTHER and UNIVERSITY are generally associated with the upper part of the frontal plane. Also namesigns which denote someone higher in the social hierarchy are also associated with an upper r-locus. This hierarchical use is 
an instance of social deixis. Social deixis marks the reference to the social characteristics of, or distinctions between, the participants in the speech event (Levinson 1983). In a signed event, social deixis is represented with r-loci established on the upper frontal plane.

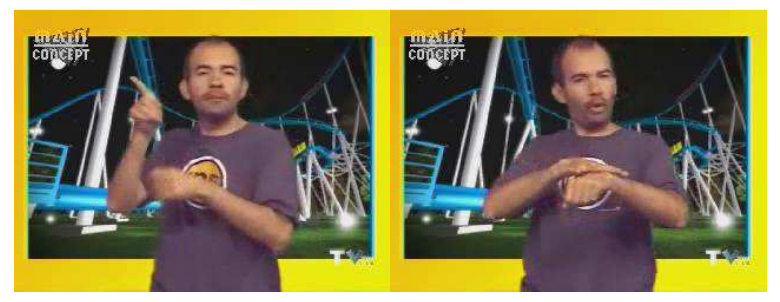

IX3 GOVERNMENT

Figure 10. Denotation of hierarchical relations

Within this use, only definite NPs referred by pronouns and namesigns (i.e. signs used as proper names within the deaf community) are localised on the upper frontal plane. In fact, this is a crucial difference with another use discussed below that denotes nonspecificity and which is only operative when localising indefinite NPs (see section 4.2.3).

\subsubsection{Locatives}

Locative NPs denote spatial locations, such as places, cities, regions and physical locations. In LSC they are usually accompanied with an index sign (Quer et al. 2005). This index sign tends to be localised on the upper frontal plane when denoting countries and bigger regions. Locative NPs are thus generally directed to an upper part of the frontal plane, both for singular (Figure 11a) and for plural DRs (Figure 11b). It is interesting to note that plural indexes functioning as locatives mark correlative points in space, rather than arc-shaped movements, which are characteristic of pronominal forms. In some contexts denoting areas within a small region or a city, the imaginary map can be extended on the horizontal plane too. 


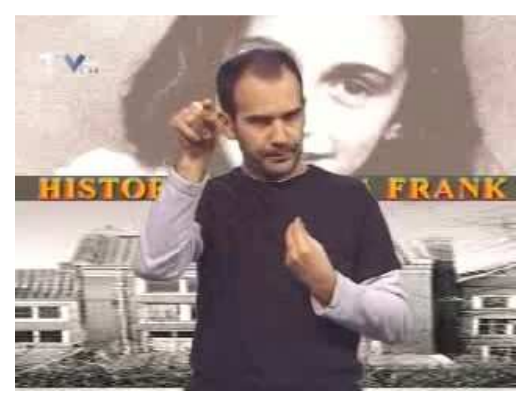

a. Singular locative

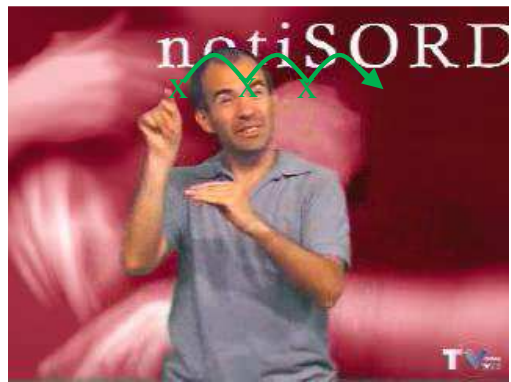

b. Plural locative

Figure 11. Locative signs directed towards the upper frontal plane

When more than one locative is used in a fragment of discourse, they are localised on the frontal plane, which is used as if it were a map, and the distance between the places and the location is considered to be at a certain scale on the plane. This use is reminiscent of the absolute localisation where real-world locations are transferred to signing space.

\subsubsection{Specificity}

The two parts of the frontal plane are also used when the signer wants to convey the specificity of the DR. The English indefinite determiner $a$ is used both for specific and non-specific NPs. The first sentence in (8) has two possible readings: a specific and a non-specific one. Yet specificity in English has observable effects on co-reference, and the resumptive pronoun disambiguates the two possible readings (Partee 1970). Under the specific reading, the indefinite NP refers to an identifiable book (8a). Under the nonspecific reading, Celia is looking for an element of the kind "syntax book", but there is not any particular book that the sender has in mind when uttering $(8 b)$.

(8) Celia wants to read a book about syntax...

a. but she cannot find it.

b. but she cannot find one.

The denotation of the same nominal localised on the upper and the lower frontal plane results in different interpretations, showing that specificity is overtly marked in LSC (Barberà 2012). A NP localised on the lower part of the frontal plane is interpreted as specific (9), which means that the sender has a particular woman in mind while uttering it. The DR is known by the sender but not by the addressee. In contrast, the nominal 
localised on the upper part is understood as non-specific (10), which means that neither the sender nor the addressee have a particular woman in mind while uttering the sentence. A subsequent utterance with a resumptive pronoun is infelicitous as shown with the symbol \# in (10).

\section{IX1 INTERVIEW IX3 $3_{1}$ WOMAN. IX3 ${ }_{1}$ SMART.}

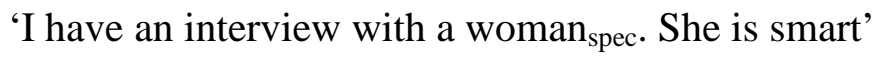

(10) IX1 INTERVIEW IX3 ${ }_{\mathrm{u}}$ WOMAN. \#IX3 ${ }_{1}$ SMART.

'I have an interview with a woman ${ }_{\text {nonspec. }}$ \#She is smart'

Another example is shown in the LSC minimal pair below. While (11) refers to a specific referent, (12) refers to a non-specific, non-identifiable one. The sentences below are graphically represented with the stills corresponding to the determiners. When the r-locus is established on the lower part of the frontal plane, it overtly expresses a specific DR (Figure 12), while an r-locus established on the upper part is circumscribed to non-specific DRs (Figure 13).

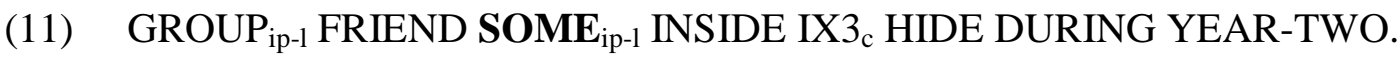

'Some of the friends were hidden there for two years.'

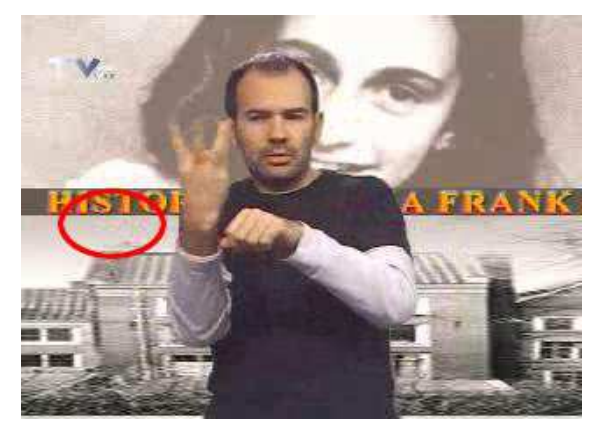

Figure 12. Lower r-locus denoting specificity

(12) IX3.pl ip-u $_{\text {u }}$ SOME 1-DENOUNCE-3 $3_{\text {ip-u }}$ IX3-c THERE-IS.

'Someone denounced they were there.' 


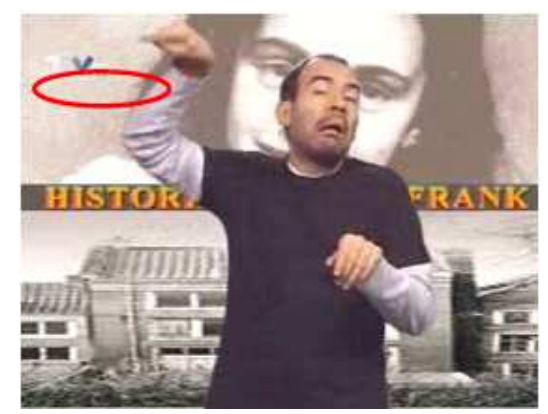

Figure 13. Upper r-locus denoting non-specificity

Importantly, the non-specific use is distinguished from the hierarchical use presented in section 4.2.1, since only NPs which are interpreted as indefinite (i.e. not being part of the common ground) are marked for non-specificity. In contrast, when denoting hierarchical relations, definite NPs such as namesigns, pronouns and definite descriptions are used to localise the corresponding entity. The difference between these two denotations is marked with non-manuals co-articulated in non-specific contexts. Indefiniteness in LSC is marked with sucking the cheeks in and pulling the mouth ends down (Figure 13 and Figure 14).

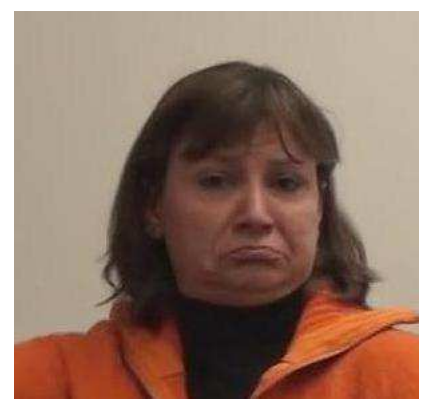

Figure 14. Indefiniteness non-manual marking

In some contexts, two different uses of the frontal plane denoting different meanings may conflate. This is the case when, for instance, a lower r-locus expressed in one element is conflated in the same NP with an upper r-locus expressed in another element. In such cases two opposed r-loci are established, although minimised for phonological reasons. That is, the lower r-locus tends to be marked loosely and with a tendency towards an upper r-locus. In (13) the determiner denotes a specific DR and hence has a direction towards the lower part. The nominal denotes a DR higher in the social hierarchy, which is commonly localised on the upper part. The two opposed directions 
are marked, although the upper direction of the nominal starts before the onset of the realisation of the nominal.

\section{UNIVERSITY $_{\mathrm{u}}$ SOME $_{1}$ EVENT PARTICIPATE.}

'Some of the universities participated in the event.'

\subsubsection{Absence in the physical context}

A final use of the frontal plane is the one that denotes absence of the DR within the immediate physical context. This is especially notorious in LSC when the [+human] entity is a DR not present in the conversation environment. Namesigns but also common nouns used to refer to someone who is not around co-occur with an index sign pointing towards the upper part of the frontal plane. In the case of common nouns, the index sign co-occurring with the noun is not articulated with the characteristic nonmanuals denoting indefiniteness (Figure 15). The lack of this non-manual articulation disambiguates the upper localisation denoting absence in the immediate physical context, rather than non-specificity.

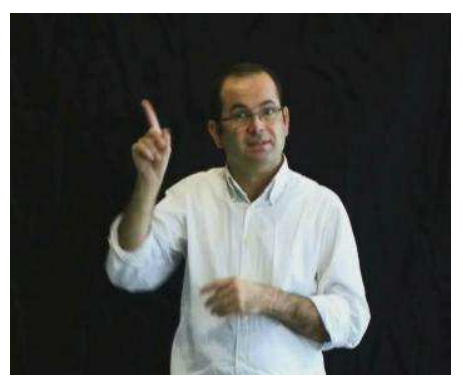

a. Reference to absent discourse referent

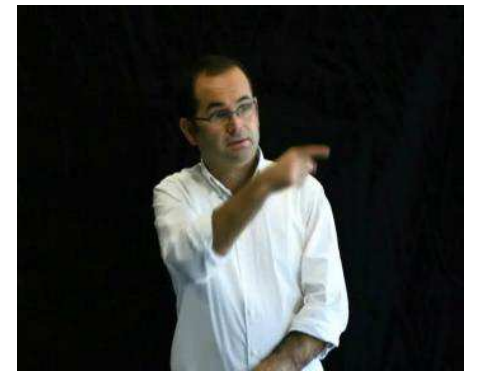

b. Reference to present discourse referent

Figure 15. Absence and presence distinction

As shown so far, the uses of the upper part of the frontal plane in LSC split into four main functions. First, it is the area where hierarchical relations are distinguished. Second, it is the place where locative signs are mainly directed. Third, non-specificity marking is overtly expressed when DRs are established in this area. And fourth, nonpresence in the immediate physical context, especially when denoting human individuals, is also marked with an index sign towards the upper part. Importantly, it has been shown that $r$-loci established on the upper frontal plane may denote four different meanings. 


\subsection{Midsagittal: Lack of distinctive discourse functions}

This final section is devoted to the description of discourse functions in the midsagittal plane, or to be more precise, to the lack of distinctive discourse functions. While the features found on the horizontal and the frontal planes have different discourse functions, this is not the case with the midsagittal plane. The distinction proximal-distal, found in the lexicon and in the morphosyntax of LSC especially to denote temporal information, is not relevant when establishing DRs in signing space. Entities are not abstractly established in LSC in the proximal as opposed to the distal part. Rather, the midsagittal plane is used as a single extension and no distinguishable areas can be established when localising referents. Thus a singleton feature [front] is distinguished and a r-locus is established in it without conveying further distinctions. ${ }^{5}$

However, when a demonstrative sign is used deictically and it points to an object present in the physical environment there is a conflation of a descriptive use of space (i.e. because of the deictic component) and a non-descriptive use. The direction of the demonstrative pointing towards a present object is always precisely oriented towards the direction where the present object is found in the physical environment. This descriptive use is conflated with a non-descriptive one, since once the entity is established in the discourse it is possible to refer back to it. As already mentioned, descriptive uses of space are freer and categorical distinctions are established with difficulty. Due to the descriptive component of these conflated structures, no distinction between [proximal] and [distal] can be straightforwardly made. This is why in non-descriptive uses the midsagittal plane is described as a single extension where no further distinctive functions are found in discourse.

\subsection{Features and discourse functions}

This final section provides a one-to-one correlation between cluster of features characterising r-loci and the corresponding discourse function. R-loci may be formed by combinations of clusters of features, since various spatial features are found: three features on the horizontal plane $\{[i p s i]$, [contra], [centre] $\}$; two features on the frontal

\footnotetext{
${ }^{5}$ A reviewer proposes a proximal vs. distal distinction in the midsaggital plane, as attested for ASL in Taub (2001). According to our data set, LSC shows a language-specific difference with respect to ASL, since DRs are not abstractly established in the proximal as opposed to the distal areas within the midsaggital plane. These results are based on a strong tendency attested in our small-scale corpus data. Still a follow-up study based on a yet non-existent large LSC corpus should help confirm the generalisations and the analysis provided here on the basis of naturalistic data.
} 
plane $\{[l o w]$ and $[u p]\}$, and one feature on the midsagittal plane [front]. Although these six combinations could potentially be the spatial areas used in the grammar, in LSC only five directions formed by clusters of features are indeed found in discourse. The [front] area on the midsagittal plane is not further divided into [low] and [up] parts, but it is rather considered to be one and only direction without further division. As for the frontal plane, the two features [low] and [up] can be combined with the lateral horizontal features, namely [ipsi] and [contra] features. That is, the [ipsi] feature can be combined with [low] and [up], and the [contra] feature can be combined with [low] and [up]. According to our data, the following combinations are possible.
a. $\{[$ front $]$, [ipsi], [low $]\}$
b. $\{[$ front $]$, [ipsi], [up]\}
c. $\{[$ front $]$, [centre $]\}$
d. $\{[$ front $]$, [contra], [low $]\}$
e. $\{[$ front $]$, [contra], [up] $\}$

These five clusters of features represent the directions where an r-locus may be established. However, no grammatical difference has been found in LSC in using [ipsi] and [contra] features apart from motivations due to assimilation processes and economy reasons. Concerning the lateral parts, what matters is the establishment of the two opposing sides, rather than the particular side of localisation. Hence, although five directions are possible, only three clusters of features are relevant in LSC grammar. The reason is that the cluster formed by $\{[$ front], [ipsi], [low] $\}$ is the mirror image of $\{[$ front $]$, [contra], [low] $\}$, and the cluster $\{[$ front $]$, [ipsi], [up] $\}$ is the mirror image of $\{[$ front $]$, [contra], [up]\}.

$$
\begin{aligned}
& \{[\text { front }], \text { [ipsi] },[\text { low }]\} \equiv\{[\text { front }], \text { [contra }],[\text { low }]\} \\
& \{[\text { front }],[\text { ipsi] },[\text { up }]\} \equiv\{[\text { front }],[\text { contra }],[\text { up }]\}
\end{aligned}
$$

While the features [ipsi] and [contra] do not imply any contrastive difference in the grammar of LSC, the [up] and [low] contrast in the frontal plane does imply a grammatical distinction. As detailed in 3.3.2, NPs localised on the upper part are associated with some particular and marked meanings, while the lower part is the 
default marking. Whereas the two features on the frontal plane are relevant and play a very specific role in LSC grammar, this is not the case for the lateral features. Three clusters of features are relevant for LSC and characterise an r-locus. Moreover, the analysis to our small-scale LSC corpus shows that they are each specialised in the contribution of referential aspects and discourse functions, as indicated below:

(16) a. $\{[$ front], [ipsi]/[contra], [low] $\}$ : specific discourse referents and contrastive topics

b. $\{[$ front], [ipsi]/[contra], [up]\}: hierarchical relations, locative information, non-specificity, absence in the physical context

c. $\{[$ front $]$, [centre] $\}$ : non-entities

\section{Conclusions}

This article has offered a description of the uses and functions of spatial planes to express discourse categories, which contributes to the characterisation of the abstract import of signing space. According to our small-scale data set, a categorical division into different directions of a three-dimensional area such as signing space is available, due to the systematic use of spatial planes in LSC with respect to their function in the discourse. The present description shows that besides the modality differences between spoken and signed languages, the features within spatial planes constitute a finite set of elements and the discourse use of r-loci in LSC can be discretely distinguished. The main aim of this article has been to develop a qualitative study by observing and analysing the tendencies that naturalistic, semi-spontaneous and elicited data from our small-scale LSC corpus provide. The results of this data set should be taken as a strong tendency of real data which is associated with discourse functions. A follow-up study based on a yet non-existent large LSC corpus should help confirm the generalisations and the analysis provided here on the basis of naturalistic data.

In this article, new insights to the ongoing debate over the nature of space in the signed modality in terms of linguistic properties have been offered. It constitutes a first step towards the characterisation of the study of spatial planes in discourse use, which needs to be contrasted with other sign languages in order to expand our cross-linguistic knowledge specifically in the discourse domain. Also, the spatial expression of scalar values and gradability, on the one hand, and the interaction between descriptive and 
non-descriptive uses of signing space, on the other, opens up an interesting avenue of research that needs to be further explored both intra and inter-language studies.

\section{Acknowledgments}

I want to express my sincerest gratitude to Josep Quer, Santiago Frigola, an anonymous reviewer and the audience at FEAST Venice 2011 for insightful comments on the main ideas of the present work. I am also very much indebted to the deaf LSC signers who participated in the data collection used for the present work. This research was partly made possible thanks to the Spanish Ministry of Science and Innovation (FFI200910492), Generalitat de Catalunya (URLING-2009SGR00763 and BE-DGR, AGAUR) and the COST Action IS1006 SignGram. I take the responsibility for any remaining mistakes.

\section{References}

Arık, E. 2009. Spatial language: Insights from signed and spoken languages. Doctoral dissertation, Purdue University.

Barberà, G. 2012. The meaning of space in Catalan Sign Language (LSC). Reference, specificity and structure in signed discourse. Doctoral dissertation. Universitat Pompeu Fabra, Barcelona.

Brennan, M. 1983. Marking time in British Sign Language. In Language in sign: An international perspective on sign language, eds. J. Kyle and B. Woll, 10-31. London: Croom Helm.

Brentari, D. 1998. A prosodic model of sign language phonology. Cambridge, MA: MIT Press.

Emmorey, K. 2007. The psycholinguistics of signed and spoken languages: How biology effects processing. In The Oxford handbook of psycholinguistics, ed. G. Gaskell, 703-721. Oxford, UK: Oxford University Press.

Engberg-Pedersen, E. 1993. Space in Danish Sign Language. The Semantics and Morphosyntax of the Use of Space in a Visual Language. Hamburg: SignumVerlag.

Fischer, S. and B. Gough. 1978. Verbs in American Sign Language. Sign Language Studies 7: 17-48.

Friedman, L. 1975. Space, Time, and Person Reference in American Sign Language. Language 51(4): 940-961. 
Janis, W. D. 1992. Morphosyntax of the ASL verb phrase. Doctoral dissertation, State University of New York at Buffalo.

Karttunen, L. 1976. Discourse referents. In Syntax and Semantics: Notes from the Linguistic Underground, ed. J. McCawley, 363-386. Academic Press: New York.

Kegl, J. 1976/2003. Pronominalization in American Sign Language. Sign Language and Linguistics 6:245-265.

Klima, E. and U. Bellugi. 1979. The signs of language. Cambridge, MA: Harvard University Press.

van der Kooij, E. 2002. Phonological categories in Sign Language of the Netherlands: The role of phonetic implementation and iconicity. Doctoral dissertation, Universiteit Leiden. Utrecht: LOT.

Levinson, S. 1983. Pragmatics. Cambridge, England: Cambridge University Press.

Levinson, S. 1996. Frames of reference and Molyneux's question. In Language and Space, ed. P. Bloom et al., 109-170. Cambridge, MA: MIT Press.

Liddell, S. 1990. Four Functions of a Locus: Reexamining the Structure of Space in ASL. In Sign Language Research: Theoretical Issues, ed. C. Lucas, 176-198. Washington D.C.: Gallaudet University Press.

Liddell, S. 2003. Grammar, Gesture and Meaning in American Sign Language. Cambridge: Cambridge University Press.

Liddell, S. and R. Johnson. 1989. American Sign Language: The Phonological Base. Sign Language Studies 64:195-277.

Lillo-Martin, D. and E. Klima. 1991. Pointing Out Differences: ASL Pronouns in Syntactic Theory. In Theoretical Issues in Sign Language Research, Vol. 1: Linguistics, eds. S. Fischer and P. Siple, 191-210. Chicago: University of Chicago Press.

Marsaja, G. 2008. Desa kolok - A deaf village and its sign language in Bali, Indonesia. Nijmegen: Ishara Press.

Mathur, G. 2000. Verb Agreement as Alignment in Signed Languages. Doctoral Dissertation, MIT.

Mayol, L. 2010. Contrastive Pronouns in Null Subject Romance Languages. Lingua, 120(10):2497-2514.

Meier, R. 1990. Person Deixis in American Sign Language. In Theoretical Issues in Sign Language Research, Vol. 1: Linguistics, eds. S. Fischer and P. Siple, 175190. Chicago: University of Chicago Press. 
Morales-López, E., R. M. Boldu-Menasanch, J.A. Alonso-Rodriguez, V. Gras-Ferrer, M. A. Rodriguez-Gonzalez. 2005. The Verbal System of Catalan Sign Language (LSC). Sign Language Studies, 5(4).

Morgan, G. 1996. Spatial Anaphoric Mechanisms in British Sign Language. In S. Botley et al., ed. Approaches to Discourse Anaphora: Proceedings of DAARC96, 500-506.

Neidle, C., J. Kegl, D. MacLaughlin, B. Bahan, and R.G. Lee. 2000. The syntax of American Sign Language. Cambridge: MIT Press.

Padden, C. 1988. Interaction of morphology and syntax in American Sign Language. New York: Garland.

Padden, C., I. Meir, W. Sandler and M. Aronoff. 2010. The grammar of space in two new sign languages. In Sign languages: A Cambridge language survey, ed. D. Brentari, 570-592. Cambridge, UK: Cambridge University Press.

Partee, B. 1970. Opacity, Co-reference, and Pronouns. Synthèse, 21 (3-4):359 - 385.

Perniss, P. 2007. Space and Iconicity in German Sign Language (DGS). MPI Series in Psycholinguistics 45. Nijmegen: Radboud University.

Poizner, H., E. Klima and U. Bellugi. 1987. What the Hands Reveal about the Brain. Cambridge, MA: MIT Press/Bradford Books.

Rathmann, Christian and Gaurav Mathur. 2002. Is verb agreement the same crossmodally? In Modality and structure in signed and spoken languages, eds. R. Meier, K. Cormier and D. Quinto-Pozos, 370-404. Cambridge: Cambridge University Press.

Russell, K. and T. Janzen. 2008. The categorical nature of ASL pronoun locations. In R. M. de Quadros, ed. Sign Languages: spinning and unraveling the past, present and future (TISLR9). Petrópolis/RJ. Brazil: Editora Arara Azul.

Quer, J. et al. 2005. Gramàtica bàsica LSC. CD-ROM. Barcelona: Federació de Persones Sordes de Catalunya.

Sandler, W. 1989. Phonological Representation of the Sign: Linearity and Nonlinearity in American Sign Language. Foris, Dordrecht.

Schlenker, P. and J. Lamberton. 2012. Formal Indices and Iconicity in ASL. In Logic, Language and Meaning: 18th Amsterdam Colloquium, eds. M. Aloni et al., 1-11. Springer-Verlag, Berlin Heidelberg.

Shepard-Kegl, J. 1985. Locative relations in ASL word formation, syntax and discourse. Doctoral dissertation, MIT.

Vallduví, E. and M. Vilkuna. 1998. On Rheme and Kontrast. In The Limits of Syntax, ed. P. Culicover and L. McNally, 79-106. New York: Academic Press. 
De Vos, C. 2012. Sign-spatiality in Kata Kolok: How a village sign language in Bali inscribes its signing space. Doctoral dissertation. Nijmegen: Radboud University.

Wilbur, R. 2008. Complex predicates involving events, time and aspect: Is this why sign languages look so similar? In Signs of the time. Selected papers from TISLR 8, ed. J. Quer, 217-250. Hamburg: Signum Verlag.

Winston, E. 1995. Spatial Mapping in Comparative Discourse Frames. In Language, gesture and space, eds. K. Emmorey and J. Reilly, 87-114 Mahwah, NJ: Lawrence Erlbaum Associates.

Zeshan, U. 2000. Sign Language in Indo-Pakistan: A description of a signed language. Amsterdam: John Benjamins Publishing Company. 